\title{
BEARING CAPACITY OF FOUNDATIONS ON SAND USING THE METHOD OF SLIP LINE
}

Meen-Wah Gui

Department of Communication Engineering, Da Yeh University, No. 112, Rd. Shan-Jeau, Da-Tsuen, Chang-Hwa, Taiwan 51505, R.O.C., mwgui@ntut.edu

Muhunthan

Professor, Department of Civil and Environmental Engineering, Washington State University, Pullman, WA 99164-2910, U.S.A.

Follow this and additional works at: https://jmstt.ntou.edu.tw/journal

Part of the Civil and Environmental Engineering Commons

\section{Recommended Citation}

Gui, Meen-Wah and Muhunthan (2006) "BEARING CAPACITY OF FOUNDATIONS ON SAND USING THE METHOD OF SLIP LINE," Journal of Marine Science and Technology. Vol. 14: Iss. 1, Article 1.

DOI: $10.51400 / 2709-6998.2074$

Available at: https://jmstt.ntou.edu.tw/journal/vol14/iss1/1

This Research Article is brought to you for free and open access by Journal of Marine Science and Technology. It has been accepted for inclusion in Journal of Marine Science and Technology by an authorized editor of Journal of Marine Science and Technology. 


\section{BEARING CAPACITY OF FOUNDATIONS ON SAND USING THE METHOD OF SLIP LINE}

\section{Acknowledgements}

The work presented herein is based on a collaborative research that was initiated at Cambridge University and subsequently completed at National Taipei University of Technology. The second author acknowledges the International Fellowship Award by the US National Science Foundation that enabled the initiation of this collaborative effort with the first author. 


\title{
BEARING CAPACITY OF FOUNDATIONS ON SAND USING THE METHOD OF SLIP LINE
}

\author{
Meen-Wah Gui* and Balasingam Muhunthan**
}

Key words: bearing capacity, slip line, sand, foundation design, critical depth.

\begin{abstract}
The accurate prediction of the bearing capacity of shallow foundations on granular soils has been complicated by their stress dependent strength behavior and the surface roughness of the foundationsoil interface. These are accounted for using empirical relations in the current state of practice. In this study, the stress dependent behavior of soils is accounted for using a strength dilatancy relationship. The method of slip line has been extended to incorporate this relationship and the effects of surface roughness to derive the depth dependent bearing capacity factor $N_{q}$. The results are presented in terms of angle of friction, depth/diameter ratio, and the effect of surface roughness of the wedge/cone. Extended method of slip line has also been carried out in order to evaluate the stress dependent strength behavior of soil. The results have been compared with centrifuge test results and the comparison result shows that the variable $-\phi$ type of analysis together with the provision of soil compressibility could lead to realistic estimates of bearing capacity of foundations having depth shallower than the critical depth.
\end{abstract}

\section{INTRODUCTION}

One of the most intriguing problems in geomechanics involves the determination of the end bearing capacity of piles or their point resistance in sand. Classical theories for the determination of the pile point resistance are extensions of the rigid plasticity theory solution for surface footing. Inherent in most of these theories is the usual assumption that sand placed at uniform density has a constant strength parameter governed by the Mohr-Coulomb failure criterion $\tau_{f}=\sigma_{n}$ $\tan \phi$ where the internal angle of friction $\phi$ is constant. It has, however, been recognized for over a half a century [34] that the shear strength of sand consists of

Paper Submitted 09/01/04, Accepted 05/18/05. Author for Correspondence: Meen-Wah Gui. E-mail: mwgui@ntut.edu.

*Associate Professor, Department of Civil Engineering, National Taipei University of Technology, No. 1, Sec. 3, ZhongXiao E. Rd., Taipei, Taiwan 10608, R.O.C.

**Professor, Department of Civil and Environmental Engineering, Washington State University, Pullman, WA 99164-2910, U.S.A. frictional and dilatancy components. The dilatancy component is a nonlinear function of the state of stress in sand and its relative density. Therefore, a realistic approach for the determination of bearing capacity must take into account of the nonlinear shear strength of the sand.

Graham and Pollock [12] have used the method of slip line and the simple strength behavior proposed by De Beer [2] in which $\phi$ varied with log mean principal stress to develop bearing capacity solution for a footing founded on the surface. The magnitude of the variation depended on the initial placement density of sand, whether "loose" or "dense". Graham and Hovan [11] have incorporated the critical state model of sand behavior to account for the effects of different stress levels and placement densities into the stress characteristic solution for surface footings. Very few attempts, however, have been made to use the method of slip line to determine the end resistance of a wedge foundation or conical pile. Houlsby and Wroth [15] have highlighted the importance of the depth effect in penetration. As a pile is advanced into the soil the tip resistance will increase as a result of the additional surcharge due to overburden and the rotation of principal stresses. Meyerhof [21] proposed an extension for footings with initial burial based on the work of Prandtl [26] and Reissner [28]. He incorporated both the shear strength of the overburden and the additional rotation of principal stress. Nowartzki [24] adopted this approach in his slip line analysis to back-analyse SPT results. It led to an increment of the included angle of the fan zone around the penetration device. The slip line reverted back to the shaft once the critical penetration depth was reached [37]. No additional rotation of principal stress was allowed beyond this depth.

Very few researchers have, however, used this technique in the solution of end bearing problems; Lee [19] adopted this technique to predict his cone penetration test results, and De Simone and Sapio [31] analyzed the end bearing of pile using a reduced extent of the slip volume.

Bolton [3] has proposed a practical model of the 
relationship between strength and dilation in granular soils. This relationship recognizes that the dilatancy angle at peak strength is a function of the relative density of the soil and mean-normal effective confinement stress. Perkins and Madson [25] used the relationship to develop practical correlations for the bearing capacity of foundations in sands. Simonini [32] incorporated the same relationship into a finite element analysis to study the behavior of dense sand surrounding the pile tip.

The present paper uses the method of slip line to find the end bearing capacity of shallow wedge foundation/conical pile first using the established approach of strength by a simple constant- $\phi$ relation; it is later extended to the more realistic stress-dependent $\phi$ relation incorporating the strength model proposed by Bolton [3]. Centrifuge tests results are also presented to examine the predictions of the proposed methods.

\section{END BEARING CAPACITY OF PILES}

Conventional theories present the solution for the ultimate tip resistance $q_{u}$ of a pile in the form:

$$
q_{u}=c N_{c}+\sigma_{v} N_{q}
$$

where $c$ represents the strength intercept (cohesion) of the assumed straight line Mohr envelope and $\sigma_{v}$ is the effective vertical stress in the ground at the pile tip level. $N_{c}$ and $N_{q}$ are dimensionless bearing capacity factors related to each other by the Caquot relation $N_{c}=$ $\left(N_{q}-1\right) \cot \phi$.

Research conducted by Vesic [31] showed that the tip resistance is governed not by the effective vertical stress but by the mean normal effective stress. Accordingly, the revised form of Eq. (1) becomes:

$$
q_{u}=c N_{c}+\frac{1+2 k_{0}}{3} \sigma_{v} N_{q}
$$

in which $k_{o}$ is the coefficient of lateral earth pressure at rest. Houlsby and Hitchman [14] demonstrated that the primary dependence of cone resistance was on horizontal rather than vertical effective stress; Bolton et al., [4], however, concluded that this effect is insignificant for the type of material to be discussed below. Since $N_{c}$ and $N_{q}$ are related to each other, attention has been focused on the determination of $N_{q}$. Note that for sands $c=0$.

For purposes of the analysis reported here the bearing capacity factors are derived for a $60^{\circ}$ shallow wedged (plane strain) and $60^{\circ}$ shallow conical (axisymmetric) pile. This value was chosen so that the results are applicable to general foundation problems. A rigid cone with an angle of $\left(45^{\circ}+\phi_{\text {crit }} / 2\right)$ with the pile tip, where $\phi_{c r i t}$ is the critical angle of friction, is gener- ally assumed to exist beneath the pile tip [27]. The critical angle of friction lies around $32^{\circ}$ for most soils and this results in a rigid cone with an angle of $61^{\circ}$, as compared with the $60^{\circ}$ adopted in the current analysis. Furthermore, centrifuge tests on offshore conical spudcan foundations showed that a $60^{\circ}$ cone in the base would produce the minimum bearing capacity [29]. Therefore, an analysis of the bearing capacity factor for a $60^{\circ}$ cone can be considered to give a lower bound value to $N_{q}$. It is noted, however, that the theoretical analyses presented are general and can be used for a wedge or cone with any apex angle.

\section{METHOD OF ANALYSIS: CONSTANT $\phi$}

In the following analyses, all stresses are referred to effective stresses unless specified otherwise.

\section{Governing equations}

Figure 1a shows the schematics of an axi-symmetric pile penetrating a soil medium with adjacent rigid plastic zones. Let cylindrical polar coordinates $(r, \theta, z)$

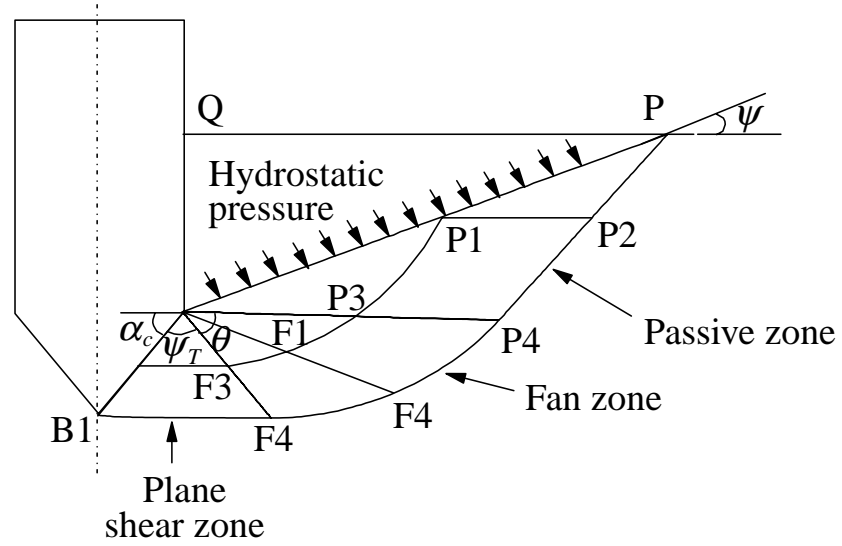

(a)

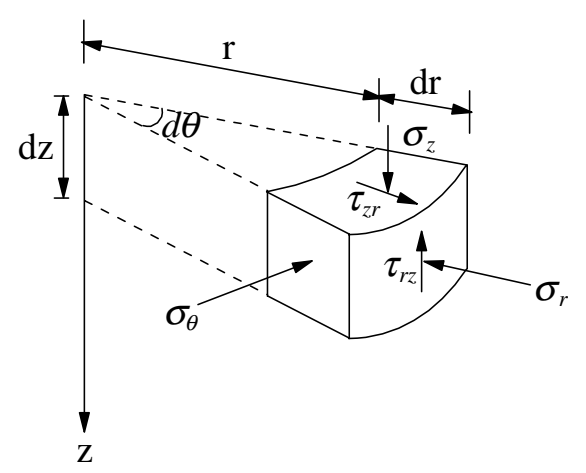

(b)

Fig. 1. (a) A very coarse stress characteristic mesh; (b) cylindrical coordinate system and stress components. 
be defined, the origin being at the center of the axis of the pile (Figure 1b). The soil occupies the semi-infinite region $z \geq 0$. In this system, the non-zero stress tensor components are $\left(\sigma_{r}, \sigma_{\theta}, \sigma_{z}, \tau_{r z}\right)$ and the body force components $(\gamma \sin \lambda, 0, \gamma \cos \lambda)$, where $\gamma$ is the magnitude and $\lambda$ is its direction.

The equations of equilibrium of the toroidal element (Figure 1b) can be written as $[17,31]$ :

$$
\begin{aligned}
& \frac{\partial \sigma_{r}}{\partial r}+\frac{\partial \tau_{r z}}{\partial z}+\frac{n}{r}\left(\sigma_{r}-\sigma_{\theta}\right)=\gamma \cdot \sin (\lambda) \\
& \frac{\partial \tau_{r z}}{\partial r}+\frac{\partial \sigma_{z}}{\partial z}+\frac{n}{r}\left(\tau_{z r}\right)=\gamma \cdot \cos (\lambda)
\end{aligned}
$$

where $n=0$ for plane strain and $=1$ for axi-symmetric problem.

The idealized soil is assumed to be rigid perfectly plastic and to obey a Mohr-Coulomb yield criterion and associated flow rule. In addition, the Haar and Von Karman [13] hypothesis is assumed to be valid and that the soil within OPQ in Figure 1a acts as a hydrostatic fluid and therefore possesses no shear strength. The Haar and Von Karman hypothesis states that the circumferential stress or hoop stress, $\sigma_{\theta}$, (Figure 1b) should be equal to either the major or the minor principal stress. Studies by Shield [30], Cox [8] and Chen [7] have shown that $\sigma_{\theta}$ is equal to the minor or the major principal stress depending on whether the soil under loading is moving outward or inward. In the case of the penetration problem, $\sigma_{\theta}$ is taken to be equal to the minor principal stress $\sigma_{3}$ because the soil medium adjacent to the shaft is moving outward during penetration. For this special case the failure condition is identical with the yield criterion, and the Mohr-Coulomb failure criterion will be used to specify the yield surface [15]. Note that it is only in the special case of perfectly plasticity that the yield and failure surfaces are identical [15]. Figure 2a shows a typical Mohr circle of a soil element at failure. The yield condition for purely frictional soils may be expressed as:

$$
\sqrt{\left(\frac{\sigma_{z}-\sigma_{r}}{2}\right)^{2}+\tau_{r z}^{2}}=\left(\frac{\left(\sigma_{z}+\sigma_{r}\right)}{2} \sin (\phi)\right)
$$

The four stress components $\left(\sigma_{r}, \sigma_{\theta}, \sigma_{z}, \tau_{r z}\right)$ can now be expressed in terms of the mean stress $s=0.5\left(\sigma_{1}\right.$ $\left.+\sigma_{3}\right)$ and the inclination angle $\psi$ subtended with the $z$ axis by the major principal stress (Figure 2b) and the friction angle $\phi$ to satisfy the yield condition:

$$
\begin{aligned}
& \sigma_{r}=s[1-\sin \phi \cos 2 \psi] \\
& \sigma_{z}=s[1+\sin \phi \cos 2 \psi]
\end{aligned}
$$

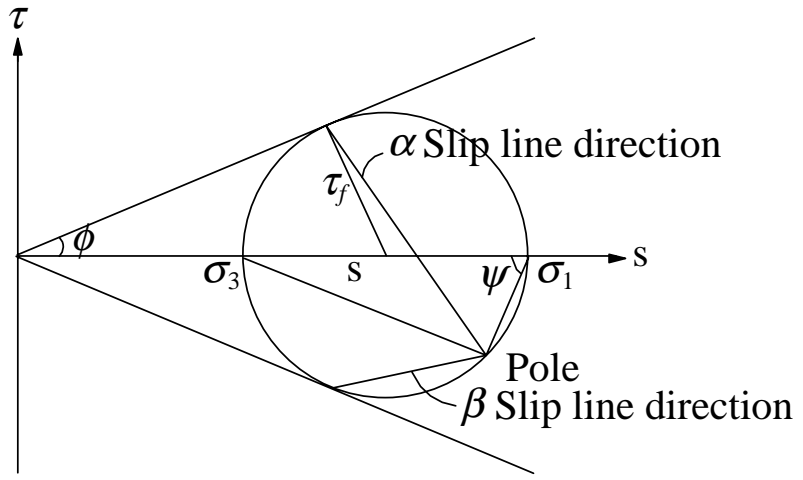

(a)

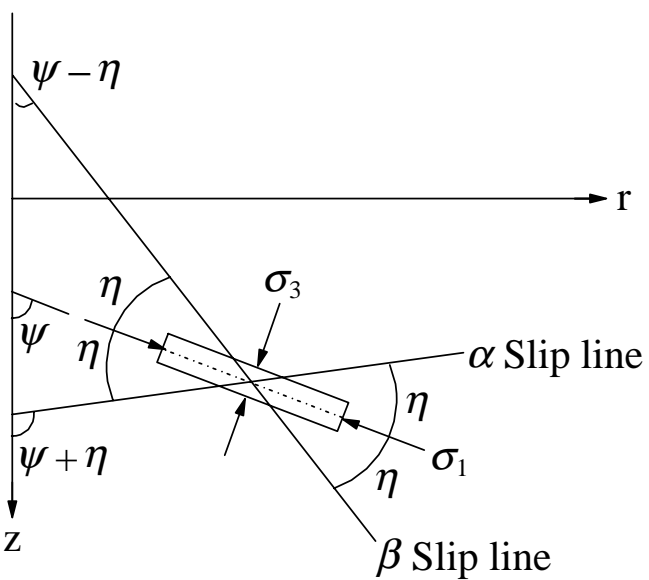

(b)

Fig. 2. (a) Mohr circle of stress under failure condition; (b) sign convention and notation for principal stresses direction.

$$
\begin{aligned}
& \sigma_{\theta}=\sigma_{3}=s[1-\sin \phi] \\
& \tau_{r z}=\tau_{z r}=s[\sin \phi \sin 2 \psi]
\end{aligned}
$$

Substitution of the above stress components into the governing equations will result in a system of nonlinear hyperbolic partial differential equations. These hyperbolic equations may be solved using the method of slip line as applied by Sokolovski [33] to many problems in plane strain and Cox et al. [9] to a problem of axial symmetry. The characteristics that will enable the solution to the set of hyperbolic equations are lines $\alpha$ and $\beta$ on which $\phi$ is mobilised. The slopes of these lines can be written as:

$$
\frac{d r}{d z}=\tan (\psi \pm \eta) \quad \text { where } \quad \eta=\frac{\pi}{4}-\frac{\phi}{2}
$$

The $\alpha$-line corresponds to the "+" value and $\beta$-line corresponds to the "-" value (Figure $2 b$ ). The equilibrium equations reduce to two ordinary differential equations along these characteristic lines, which after rear- 
ranging becomes:

$$
\begin{aligned}
& d s \pm 2 s(\tan \phi) d \psi+n \frac{s}{r \cos \phi}[\sin \phi \cos \phi d r \\
& \left. \pm\left(\sin ^{2} \phi-\sin \phi\right) d z\right] \\
& =\frac{\gamma}{\cos \phi}[\sin (\lambda \mp \phi) d r+\cos (\lambda \mp \phi) d z]
\end{aligned}
$$

The equation corresponding to the upper sign holds along the $\alpha$-characteristics while that corresponding to the lower sign holds along the $\beta$-characteristics. $n$ is taken to be zero in plane strain analysis while it is unity for axi-symmetric analysis [17].

The form of Eq. (7) is complex for an analytical solution. Therefore, a numerical solution using a finite difference scheme is sought. In addition, the variables have to be normalized according to some characteristic length [33] to obtain the solution of the governing equation. Following Larkin [17] the variables are normalized to a scale length $0.5 B$ by defining:

$$
\sum=\frac{s}{0.5 \gamma B} ; R=\frac{r}{0.5 B} ; \text { and } Z=\frac{z}{0.5 B}
$$

where $B$ is the width or diameter of foundation. Eq. (7) can now be written in finite difference form, suitable for the solution of the intersection of an $\alpha$-line, which passed through a known point $\left(R_{1}, Z_{1}, \Sigma_{1}, \psi_{1}\right)$, and a $\beta$ line, which passed through another known point $\left(R_{2}, Z_{2}\right.$, $\Sigma_{2}, \psi_{2}$ ). Accordingly, Eq. (7) becomes:

$$
\begin{aligned}
& \left(\Sigma-\Sigma_{1}\right)+2 \Sigma_{1} \tan \phi\left(\psi-\psi_{1}\right) \\
& =\frac{-2 n \Sigma_{1}}{R_{1} \cos \phi}\left[\sin \phi \cos \phi\left(R-R_{1}\right)+\left(\sin ^{2} \phi-\sin \phi\right)(Z\right. \\
& \left.\left.-Z_{1}\right)\right]+\frac{1}{\cos \phi}\left[\sin (\lambda-\phi)\left(R-R_{1}\right)\right. \\
& \left.+\cos (\lambda-\phi)\left(Z-Z_{1}\right)\right]
\end{aligned}
$$

and

$$
\begin{aligned}
& \left(\Sigma-\Sigma_{2}\right)+2 \Sigma_{2} \tan \phi\left(\psi-\psi_{2}\right) \\
& =\frac{-2 n \Sigma_{2}}{R_{2} \cos \phi}\left[\sin \phi \cos \phi\left(R-R_{2}\right)-\left(\sin ^{2} \phi-\sin \phi\right)(Z\right. \\
& \left.\left.-Z_{2}\right)\right]+\frac{1}{\cos \phi}\left[\sin (\lambda+\phi)\left(R-R_{2}\right)\right. \\
& \left.+\cos (\lambda-\phi)\left(Z-Z_{2}\right)\right]
\end{aligned}
$$

For a body force acting vertically downward $(\lambda=$ 0), following Shi [29], Eq. (9a) and Eq. (9b) can be reduced to a compact form using the following substitutions:

$$
\begin{aligned}
& A=\frac{-2 n \Sigma_{1}}{R_{1} \cos \phi}\left[\sin \phi \cos \phi\left(R-R_{1}\right)\right. \\
& \left.+\left(\sin ^{2} \phi-\sin \phi\right)\left(Z-Z_{1}\right)\right]-\left[\tan \phi\left(R-R_{1}\right)+\left(Z-Z_{1}\right)\right] \\
& +\Sigma_{1}+2 \Sigma_{1}(\tan \phi) \psi_{1} \\
& B=\frac{-2 n \Sigma_{2}}{R_{2} \cos \phi}\left[\sin \phi \cos \phi\left(R-R_{2}\right)\right. \\
& \left.-\left(\sin ^{2} \phi-\sin \phi\right)\left(Z-Z_{1}\right)\right]+\left[\tan \phi\left(R-R_{1}\right)+\left(Z-Z_{2}\right)\right] \\
& +\Sigma_{2}+2 \Sigma_{2}(\tan \phi) \psi_{2}
\end{aligned}
$$

where $A=\Sigma+2 \Sigma_{1}(\tan \phi) \psi$ and $B=\Sigma-2 \Sigma_{2}(\tan \phi) \psi$, so that

$$
\Sigma=\frac{A \Sigma_{2}+B \Sigma_{2}}{\sum_{1}+\Sigma_{2}} \text { and } \psi=\frac{B-A}{-2 \tan \phi\left(\Sigma_{1}+\Sigma_{2}\right)}
$$

The above equation along with the following finite difference forms of Eq. (6)

$$
\begin{aligned}
& R-R_{1}=\left(Z-Z_{1}\right) \tan \left(\psi_{1}+\eta\right) \text { and } \\
& R-R_{2}=\left(Z-Z_{2}\right) \tan \left(\psi_{2}+\eta\right)
\end{aligned}
$$

are used in the iteration for the solution.

\section{Organization of computation}

The computational sequence for the above analysis is shown in the form of a flow chart in Figure 3. A point within the plastic field can be determined by solving Eq. (11) and Eq. (12). Referring to Figure 4, suppose that the boundaries for point $P\left(R_{P}, Z_{P}, \psi_{P}, \Sigma_{P}\right)$ and $Q\left(R_{Q}, Z_{Q}, \psi_{Q}, \Sigma_{Q}\right)$ are known. $R$ and $Z$ for point $W$ can be calculated from Eq. (12) by putting $\psi_{1}=\psi_{P}$ and $\psi_{2}=\psi_{Q}$. Subsequently, $\psi$ and $\psi_{W}$ can be determined from Eq. (11). Since most of the characteristic lines are curved $\psi$ will not be equal to $\psi_{W}$ and $\Sigma$ will not be equal to $\Sigma_{W}$. In order to improve the solution, $\psi_{1}$ and $\psi_{2}$ in Eq. (11) were initially approximated to $\left(\psi+\psi_{P}\right) / 2$ and $(\psi+$ $\left.\psi_{Q}\right) / 2$ respectively. This process was repeated until $\psi$ and $\Sigma$ converged [33]. The iteration in this study was stopped when a convergence criterion of $0.01 \%$, was achieved.

Figure 1a shows a very coarse mesh generated when a wedge pile is advanced below the ground level. The complete marching scheme adopted in the computer program for the plane strain slip line of this kind is as shown below:

(1) Passive zone - the boundary conditions at $\mathrm{O}, \mathrm{P} 1$ and 
$\mathrm{P}$ are known. $\psi$ is assumed to be in the direction of OP. P2 can be found by using the boundary conditions of $\mathrm{P}$ and $\mathrm{P} 1$ and $\mathrm{P} 3$ determined from known boundary of $\mathrm{O}$ and $\mathrm{P} 1$. $\mathrm{P} 4$ can be found by using the boundary conditions of P2 and P3. O, P3 and P4 have now become the known boundary conditions for the radial fan zone.

(2) Degenerated radial fan zone - point $\mathrm{O}$ is known as Prandtl singularity. Being a singularity, $\mathrm{O}$ can have the same $\mathrm{R}$ and $\mathrm{Z}$, but different $\psi$ and $\Sigma$. Furthermore,

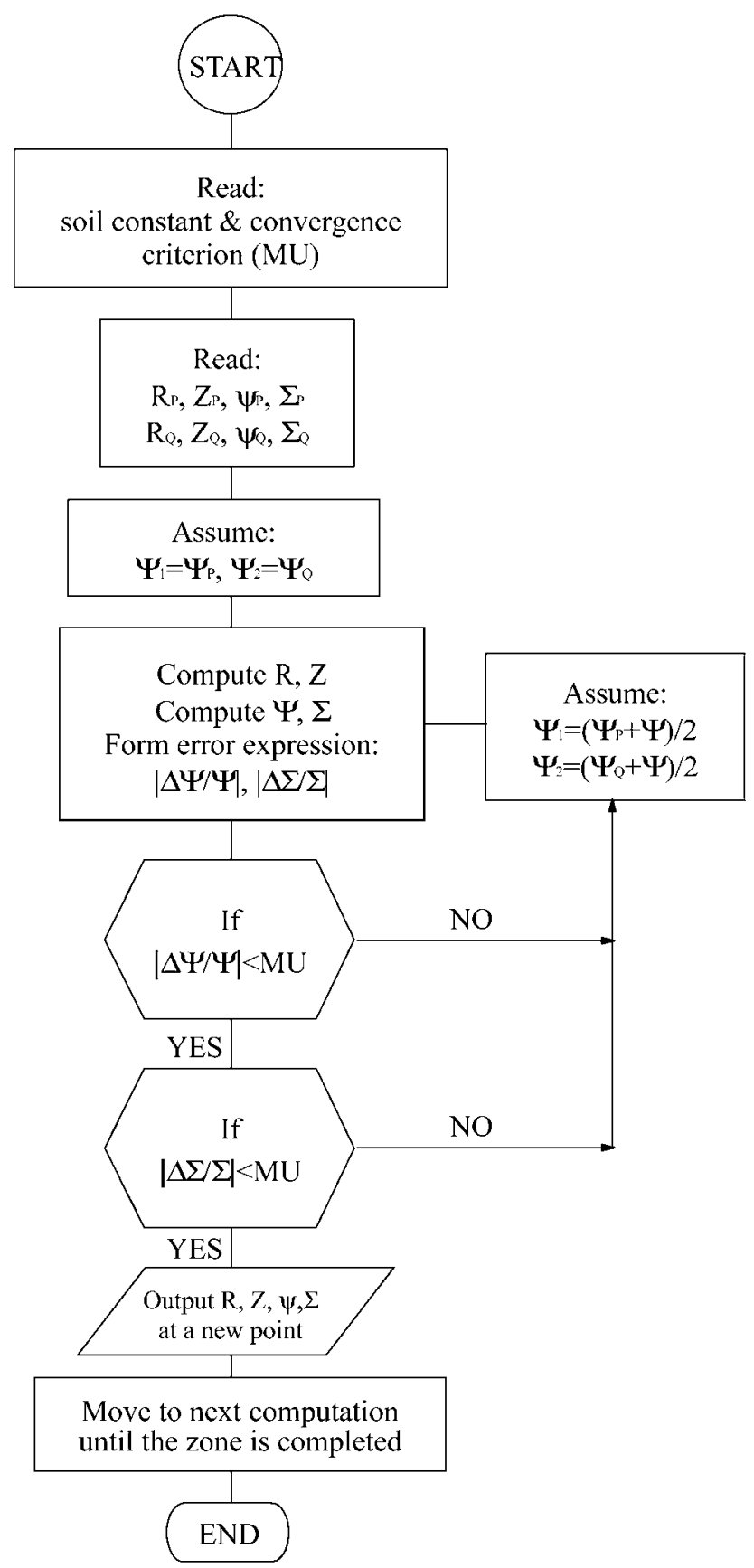

Fig. 3. Flow chart for the computation of $\boldsymbol{R}, \boldsymbol{Z}, \psi$ and $\Sigma$. within the fan zone, the stress will increase exponentially in accordance with the amount of rotation of the principal stress direction. Hence, when the principal stress rotates by $\theta$ at $\mathrm{O}$ the boundary condition becomes

$$
\Sigma_{(\theta)}=\Sigma \exp (2 \theta \tan \phi) ; \text { and } \psi_{(\theta)}=\psi+\theta
$$

The angle $\theta$ can be calculated if the roughness of the cone is given. By knowing this angle, the radial fan zone can be subdivided into several equal-angle fans so that $\Sigma_{(\theta)}$ and $\psi_{(\theta)}$ can be determined. For example, if $\theta=\theta / 2, \mathrm{~F} 1$ can be found if $\mathrm{O}_{(\theta / 2)}$ and $\mathrm{P} 3$ are known. Subsequently, F2 can be obtained from F1 and P4. When $\theta=\theta, \mathrm{O}_{(\theta / 2)}$ and $\mathrm{F} 1$ will become the boundary conditions for F3. O, F3 and F4 have now become the boundary conditions for plane shear zone.

(3) Plane shear zone - the angle of plane shear zone as shown later depends on the roughness of the conical surface, hence $\psi$ for any point on the conical surface is known. $\mathrm{B}$ is in direct contact with the cone and $\psi_{\mathrm{B}}$, known as imposed boundary condition, can be used to generate $\mathrm{R}, \mathrm{Z}$ and $\Sigma$ from the known boundary condition at F3. Once B is known, B1 can be calculated from $\mathrm{B}$ and $\mathrm{F} 4$ in the usual procedure.

\section{Effect of surface roughness of wedge/cone}

The surface roughness of the end of the wedge foundation/conical pile would influence the end bearing values and must be accounted for in the analysis [19]. Lee [19] showed that such angle $\psi_{T}$ of the plane shear zone $\mathrm{OAB}^{\prime}$ in Figure 5a adjacent to the wedge/cone can be determined from the corresponding Mohr circle in Figure 5b. Assuming that the stresses acting on plane $\mathrm{OA}$ are $\left(\sigma_{a}, \tau_{a}\right)$ and $\mathrm{OB}^{\prime}$ is the slip line where the shear

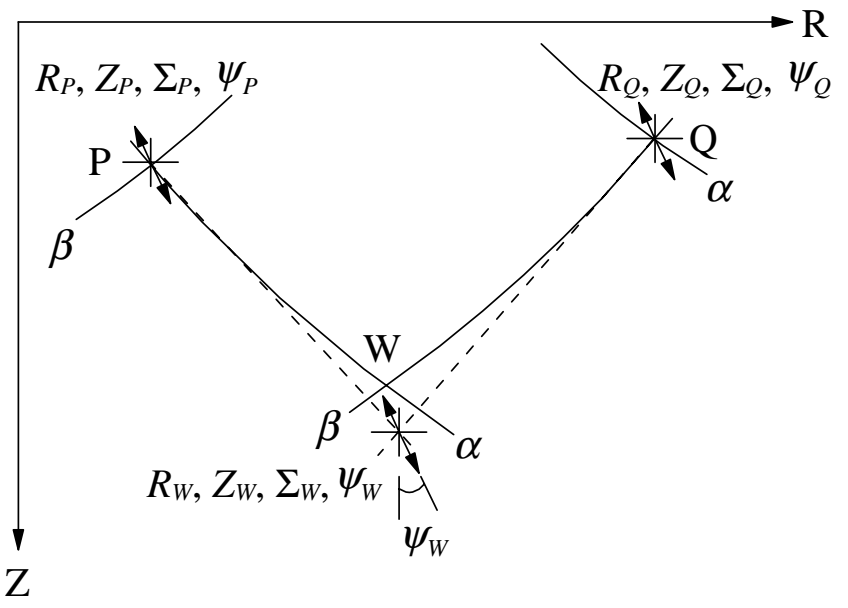

Fig. 4. Computation of a new point $W$ from two known points $P$ and $Q$. 
strength has been fully mobilized under stresses $\left(\sigma_{b}\right.$, $\left.\tau_{b}\right)$. Given that the radius of the Mohr-circle $s_{m} A^{\prime}=s_{m} B^{\prime}$ $=\left(\sigma_{1}-\sigma_{3}\right) / 2$, therefore:

$$
\begin{aligned}
& \tau_{a}=\frac{\left(\sigma_{1}-\sigma_{3}\right)}{2} \cos \left(2 \psi_{T}-\phi\right) \text { and } \\
& \sigma_{a}=\frac{\left(\sigma_{1}+\sigma_{3}\right)}{2}+\frac{\left(\sigma_{1}-\sigma_{3}\right)}{2} \sin \left(2 \psi_{T}-\phi\right)
\end{aligned}
$$

and the ratio

$$
\frac{\tau_{a}}{\sigma_{a}}=\tan \delta=\frac{\sin \phi \cos \left(2 \psi_{T}-\phi\right)}{1+\sin \phi \sin \left(2 \psi_{T}-\phi\right)}
$$

where $\delta=$ the interface friction angle. Using trigonometry identities in Eq. (15) and solving leads to a simpler equation for the angle $\psi_{T}$ :

$$
\psi_{T}=\frac{1}{2}\left[\phi-\delta+\cos ^{-1}\left(\frac{\sin \delta}{\sin \phi}\right)\right]
$$

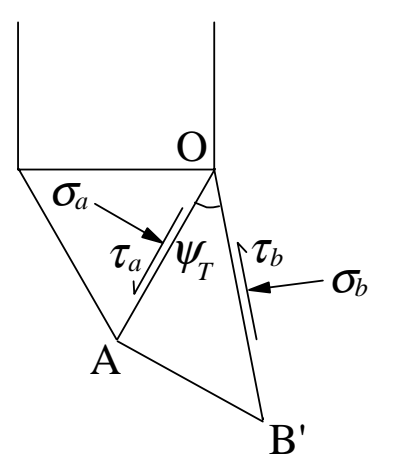

(a)

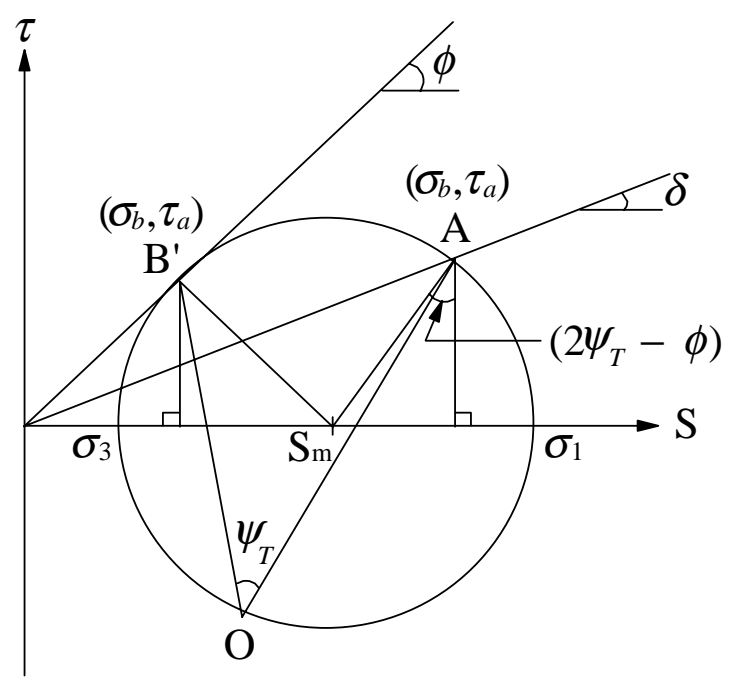

(b)

Fig. 5. (a) Component of stresses acting on a rough wedge; and (b) typical state of stress for a rough tip.
According to Meyerhof [22], as the roughness of the wedge increases, the angle $\psi_{T}$ in zone $\mathrm{OAB}^{\prime}$ decreases like the case of an inclined load on a horizontal base. For a perfectly smooth face $(\delta / \phi=0)$ and for perfectly rough face $(\delta / \phi=1)$, Eq. (16) reduces to $\psi_{T}=$ $\left(45^{\circ}+\phi / 2\right)$ and $\psi_{T}=0$, respectively.

The program iterates by assuming various values of $\mathrm{R}$ such that the $\beta$ characteristic that starts at $\mathrm{P}$ actually ends at B1 (Figure 1a). By marching from O, P1 and P to $\mathrm{O}, \mathrm{B}$ and $\mathrm{B} 1$, all the variables at $\mathrm{O}, \mathrm{B}$ and $\mathrm{B} 1$ are known. Knowing the mean stress $s=\Sigma \times 0.5 \gamma B$ at each point and the direction of major principle stress $\psi$, stress components $\sigma_{r}, \sigma_{z}, \tau_{r z}=\tau_{z r}$ and $\sigma_{\theta}$ at each point can be obtained by back substitution into Eq. (5a) through Eq. (5d). It must be noted that the stress distribution on the cone/wedge face is not constant, thus following Lee [19], the normal stress at each subdivided point on the cone/wedge with different surface roughness may be given by:

$\sigma_{n i}=\frac{\sigma_{r i}+\sigma_{z i}}{2}+\cos \left(90^{\circ}+\phi-2 \psi_{T}\right) \sqrt{\left(\frac{\sigma_{r i}-\sigma_{z i}}{2}\right)^{2}+\tau_{r z i}^{2}}$

where $i$ is the point number. The normal force is then determined from the average of the normal stress of two adjacent points, their spacing and radii from the central axis. Finally, the penetration resistance can be computed from the equilibrium of forces [19]

$$
q_{u}=\frac{4}{\pi B^{2}} \frac{\cos \left(\psi_{c}-\delta\right)}{\cos \delta} \sum_{i=1}^{i} F_{n i}
$$

where $F_{n i}$ is the normal force acting on each element bounded by two points.

\section{Computation of $N_{q}$}

The scheme developed above enables the solution of the bearing capacity of a foundation taking surcharge and soil self-weight simultaneously into consideration. According to Terzaghi [35], if the bearing capacity of a foundation on weightless frictional soil due to surcharge $\sigma_{o}$ could be calculated and expressed as $\sigma_{o} N_{q}$, and if the bearing capacity of the same foundation due to self-weight $\gamma$ alone could be written $0.5 \gamma \mathrm{BN} \gamma$, then these components could safely be superposed. Davis and Booker [10] and Bolton and Lau [6] performed rigorous checks on this superposition assumption for the plane strain and circular footings, respectively. $N_{q}$ is obtained by applying a surcharge $\sigma_{o}$ to the plane surface of the soil around the foundation. Following Eq. (8), the surcharge variable needs to be normalized be a 
scale length 0.5B, thus Bolton and Lau [6] introduced a new dimensionless parameter termed the superposition factor:

$$
\Omega=\frac{\sigma_{o}}{0.5 \gamma B}
$$

Following Bolton and Lau [6], $N_{q}$ is then obtained by increasing the value of $\Omega$ until it has no significant influence on the final result (in this case $\Omega$ was found to equal 1,000). The accuracy of this technique depends on the number of the characteristic lines and the error criterion. For error criterion of $0.01 \%$, it was found that bearing capacity factors converged when a mesh comprising $100 \beta$-characteristics and $280 \alpha$-characteristics and the degenerated radial fan zone divided into 50 small radial fans for both rough and smooth surfaces.

The distribution of pressure and slip lines for the plane strain (wedge) penetration of a pile at a specific depth is shown in Figure 6a and that corresponding to an axi-symmetric (cone) penetration at the same depth is shown in Figure $6 \mathrm{~b}$. It can be seen that the roughness of the pile surface affects the shape of the slip surface
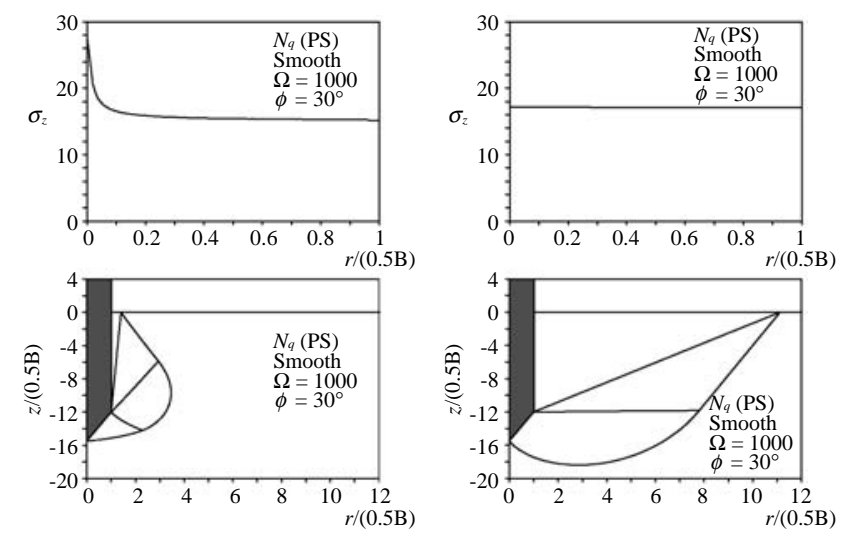

(a)
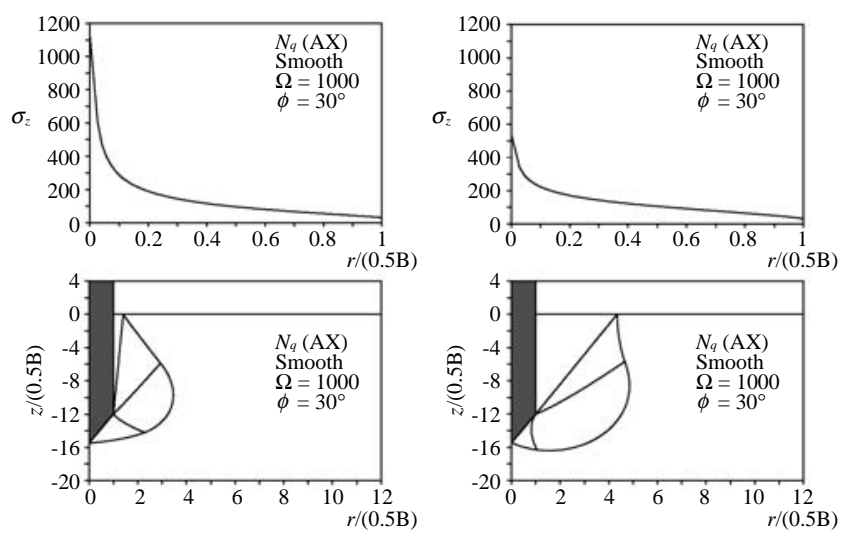

(b)

Fig. 6. Pressure and stress characteristic acting on a (a) wedge; and (b) cone. mesh very much. The effect is seen to be more in the case of the plane strain penetration. The continuous reversion of slip lines with increase in penetration depth is shown in Figure 7.

The results of the calculation of the $N_{q}$ values with depth for the different conditions are tabulated in Table 1. The conditions imposed are: plane strain or axisymmetric case, different depth ratios $\mathrm{Z}$, and different values of surface roughness ratio $\delta / \phi=0.00,0.25,0.50$, 0.75 and 1.00 . Thus, the tabulated values have accounted for both the shape and depth factors, as usually done with the conventional bearing capacity factors. As expected the $N_{q}$ values are different for a plane strain case from that for an axi-symmetric case at the same depth. Moreover, they are affected significantly by the depth ratio $Z$ and the nature of the roughness of the wedge/conical pile surface. The values of $N_{q}$ increase with the increase of penetration depth and approach constant values after reaching the so-called critical depth (in this case $Z=z /(0.5 \mathrm{~B})=16$ ). This is considered to be sufficient as it was found that the critical depth ratio only varied between 8 and 16 for tests conducted by Bolton et al. [4] under different relative densities (between $58 \%$ to $89 \%$ ). The value of $N_{q}$ remains almost constant beyond $Z=16$, at which the bearing capacity theory no longer applies [23]. For a perfectly rough surface $(\delta / \phi=1.0)$, the value of $N_{q}$ could be as high as twice the value of $N_{q}$ for a perfectly smooth surface ( $\delta /$ $\phi=0.0)$.

\section{EXTENDED METHOD OF ANALYSIS: VARIABLE}

As discussed before, $\phi$ is strongly dependent on the mean effective stress. Therefore, it is necessary to incorporate the stress-level dependent behaviour di-
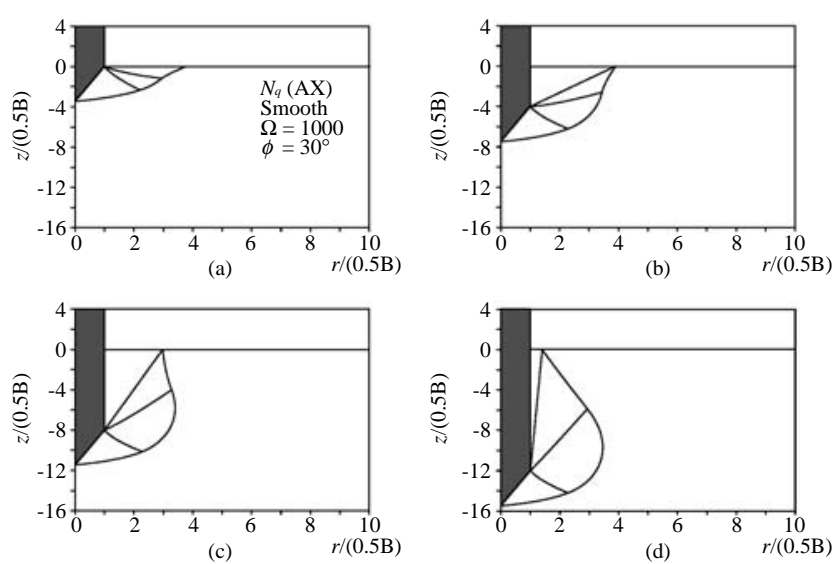

Fig. 7. Reversion of slip lines during the advancement of a smooth cone for (a) $Z=0$; (b) $Z=4$; (c) $Z=8$; and (d) $Z=12$. 
Table 1. Values of bearing capacity factor $\mathrm{Nq}$ for both axi-symmetry and plane strain cases

\begin{tabular}{|c|c|c|c|c|c|c|c|c|c|c|c|}
\hline \multirow{3}{*}{$\mathrm{Z}=z /(0.5 \mathrm{~B})$} & \multirow{3}{*}{$\delta / \phi$} & \multicolumn{5}{|c|}{ Axi-symmetry } & \multicolumn{5}{|c|}{ Plane strain } \\
\hline & & \multicolumn{5}{|c|}{$\phi$} & \multicolumn{5}{|c|}{$\phi$} \\
\hline & & 25 & 30 & 35 & 40 & 45 & 25 & 30 & 35 & 40 & 45 \\
\hline \multirow{5}{*}{0} & 0.00 & 7.8 & 12.4 & 20.5 & 35.2 & 64.1 & 4.2 & 5.8 & 8.1 & 11.8 & 18.0 \\
\hline & 0.25 & 10.8 & 18.8 & 34.1 & 65.7 & 137.5 & 5.7 & 8.6 & 13.2 & 21.3 & 36.4 \\
\hline & 0.50 & 14.1 & 26.1 & 51.0 & 107.4 & 250.4 & 7.4 & 11.8 & 19.5 & 34.0 & 63.9 \\
\hline & 0.75 & 17.4 & 33.7 & 69.7 & 156.8 & 396.7 & 9.1 & 15.2 & 26.5 & 49.4 & 100.2 \\
\hline & 1.00 & 20.2 & 40.4 & 86.4 & 202.5 & 537.7 & 10.7 & 18.4 & 33.5 & 65.3 & 140.7 \\
\hline \multirow{5}{*}{4} & 0.00 & 17.7 & 28.9 & 48.2 & 82.9 & 149.2 & 6.2 & 8.6 & 12.2 & 17.9 & 26.7 \\
\hline & 0.25 & 22.5 & 39.6 & 72.0 & 137.2 & 279.0 & 8.0 & 12.1 & 18.6 & 29.7 & 49.7 \\
\hline & 0.50 & 27.4 & 51.3 & 99.6 & 205.3 & 460.5 & 10.0 & 15.9 & 26.1 & 44.8 & 82.1 \\
\hline & 0.75 & 32.2 & 62.9 & 128.5 & 280.9 & 679.8 & 11.9 & 19.8 & 33.9 & 62.3 & 123.6 \\
\hline & 1.00 & 36.2 & 72.5 & 152.8 & 346.6 & 876.8 & 13.7 & 23.5 & 42.2 & 80.6 & 169.0 \\
\hline \multirow{5}{*}{8} & 0.00 & 32.5 & 54.3 & 89.9 & 152.7 & 270.8 & 8.5 & 11.8 & 16.9 & 24.4 & 36.2 \\
\hline & 0.25 & 40.0 & 69.7 & 126.2 & 236.1 & 469.7 & 10.6 & 15.9 & 24.4 & 38.4 & 63.5 \\
\hline & 0.50 & 47.1 & 87.1 & 166.3 & 335.7 & 733.1 & 12.8 & 20.3 & 31.3 & 55.3 & 100.8 \\
\hline & 0.75 & 53.5 & 103.2 & 207.0 & 442.1 & 1036.9 & 15.0 & 24.8 & 42.3 & 76.1 & 147.3 \\
\hline & 1.00 & 58.7 & 116.1 & 239.6 & 530.1 & 1295.2 & 16.9 & 28.8 & 51.2 & 96.3 & 197.8 \\
\hline \multirow{5}{*}{12} & 0.00 & 46.2 & 81.2 & 145.7 & 247.5 & 427.5 & 11.7 & 15.8 & 19.6 & 30.6 & 46.3 \\
\hline & 0.25 & 59.2 & 108.9 & 198.4 & 366.3 & 708.6 & 13.8 & 20.3 & 30.7 & 47.8 & 77.9 \\
\hline & 0.50 & 70.0 & 133.5 & 254.2 & 503.3 & 1075.6 & 16.1 & 25.2 & 40.5 & 67.7 & 120.1 \\
\hline & 0.75 & 77.5 & 153.0 & 308.0 & 644.8 & 1474.6 & 18.4 & 30.1 & 50.9 & 90.2 & 171.7 \\
\hline & 1.00 & 85.8 & 172.5 & 350.0 & 756.7 & 1801.3 & 20.5 & 34.6 & 60.6 & 112.5 & 227.2 \\
\hline \multirow{5}{*}{16} & 0.00 & 54.1 & 104.3 & 198.9 & 359.7 & 641.0 & 16.7 & 20.2 & 27.5 & 38.6 & 57.1 \\
\hline & 0.25 & 72.0 & 139.3 & 282.8 & 522.3 & 1009.4 & 17.5 & 25.5 & 37.7 & 57.4 & 93.2 \\
\hline & 0.50 & 89.1 & 174.2 & 360.7 & 710.7 & 1490.8 & 20.0 & 30.7 & 48.6 & 80.2 & 128.8 \\
\hline & 0.75 & 106.2 & 210.9 & 432.2 & 892.4 & 1999.7 & 22.1 & 36.1 & 60.0 & 104.9 & 197.1 \\
\hline & 1.00 & 117.2 & 236.2 & 485.7 & 1031.3 & 2400.9 & 24.6 & 40.9 & 70.7 & 129.5 & 257.4 \\
\hline
\end{tabular}

rectly into the method of slip line presented above to obtain better estimates of $N_{q}$. An interesting approach to the shear strength of sands as a function of the soil relative density and mean normal stress has been proposed by Bolton [3]:

$$
\phi=\phi_{c r i t}+\Delta I_{R}
$$

where $\phi_{c r i t}$ is the critical state friction angle and $\Delta$ is an empirical constant having a value of 3 for triaxial conditions and 5 for plane strain conditions; $I_{R}$ was termed the dimensionless dilatancy index and for penetration problem it is written as [5]:

$$
I_{R}=I_{D} \ln \left(\frac{p_{c}}{\sqrt{\sigma_{v}^{\prime} \cdot q_{u}}}\right)-1
$$

in which $I_{D}$ is the relative density, $p_{c}$ the aggregate crushing parameter $(=42,000 \mathrm{kPa}$ for study sand $)$, and $q_{u}$ the measured tip resistance. Extension of the method of slip line with Bolton's formulation will yield the stress dependent $N_{q}$ factor.

Lau [18] presented a variable $-\phi$ analysis for the surface-footing problem; his formulation is extended here to account for pile penetration. As a result of incorporating the variable $-\phi$ analysis, two possible effects; (a) effect on the stress rotation equation, and (b) effect on the geometry of the characteristic lines, result.

\section{Effect on stress rotation}

Figure 8 a shows the major principal stress, $s_{1}$, passing through a discontinuity and being distorted by 
an angle $\theta$. The corresponding Mohr circles are shown in Figure $8 \mathrm{~b}$ where the Mohr circles of stresses on either side of the discontinuity pass through a common stress point, $D[1]$. Solving the geometry problem in Figure $8 \mathrm{~b}$ leads to:

$$
\frac{s_{1}+d s_{1}}{s_{1}}=\frac{\sin (\omega+\xi)}{\sin (\omega-\xi)}
$$

where $\xi$ is equal to the mobilized angle of shearing resistance on that plane. Hence, the change in stress conditions across a discontinuity can simply be related to the rotation of major principal stress direction. Applying sine rule to $\triangle \mathrm{ABC}$ in Figure $8 \mathrm{~b}$ :

$$
\frac{d s_{1}}{\sin 2 d \theta}=\frac{s_{1} \sin \phi}{\sin (\omega-\xi)}
$$

Also, from the $\triangle \mathrm{OXB}$, we have

$$
\omega=\sin ^{-1}\left(\frac{\sin \xi}{\sin \phi}\right)
$$

In the radial fan zone where the angle of shearing

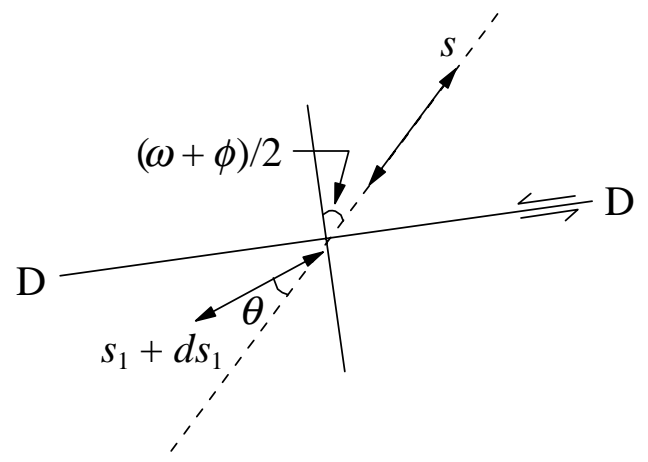

(a)

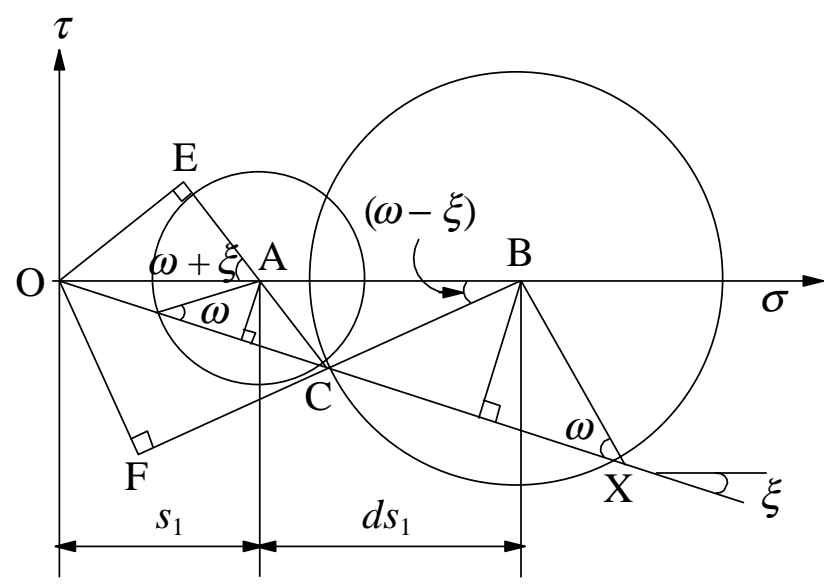

(b)

Fig. 8. Major principal stress passing through a discontinuity. has been fully mobilized, $\xi \rightarrow \phi$. In the limit as $d \theta \rightarrow 0$, $\sin (2 d \theta) \rightarrow 2 d \theta$ and Eq. (23) reduces to:

$$
\frac{d s_{1}}{s_{1}}=2 \tan \phi d \phi
$$

Figure 9 shows that geometry of a discontinuity between limiting states $\left(s_{1}, \phi\right)$ and $\left(s_{1}+d s_{1}, \phi+d \phi\right)$. Here, $d \phi / d s_{1}$ has been taken as positive merely for mathematical consistency [18]. By cosine rule and rearranging, we get the stress rotation Eq.

$$
d \theta=\sqrt{\frac{\left(\frac{d s_{1}}{s_{1}}-\tan \phi d \phi\right)^{2}-\sec ^{2} \phi d \phi^{2}}{4 \tan ^{2} \phi}}
$$

Substituting $s_{1}$ with the mean effective stress $s$, we thus obtain Eq. (26) as the general stress rotation equation. Lau [18] performed the validation of the general stress rotation equation for a surface footing on a frictionless material.

\section{Effect on geometry of slip lines}

The geometry of the characteristic lines is related to the tangency of a Mohr circle on the limiting Mohr envelope. Therefore, a second parameter $\phi_{\text {en }}$ must be defined so that the angle between the two characteristics at any point remains $90-\phi_{e n}$, where

$$
\begin{aligned}
& \phi_{e n}=\sin ^{-1}\left[\sin \phi+s \frac{d \phi}{d s} \cos \phi\right] \\
& 2 d \theta+\left(s_{1}+d s_{1}\right) \sin (\phi+d \phi)
\end{aligned}
$$

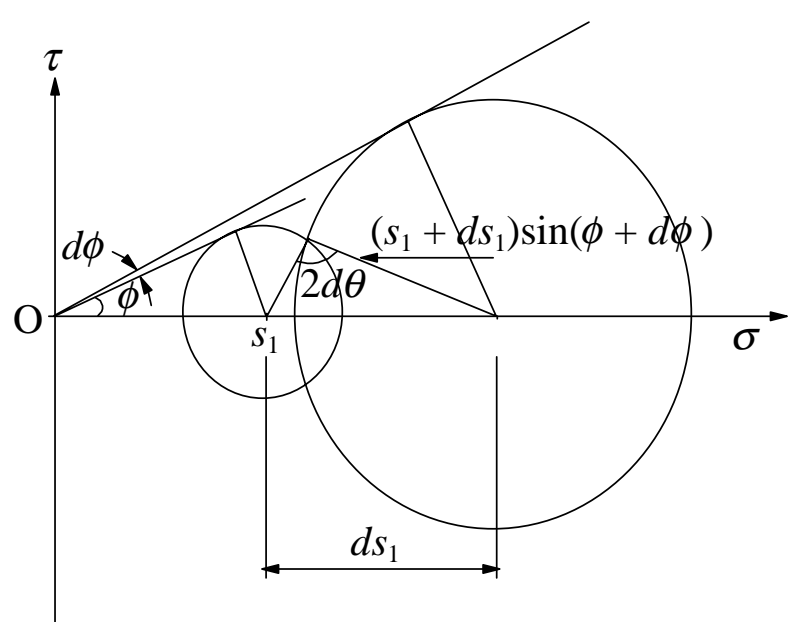

Fig. 9. Effects of varying $\phi$ on the stress rotation equation (after [18]). 
The parameter $d \phi / d s$ in the above expression can be obtained by combining Bolton's [3,5] relationships, Eq. (20) and Eq. (21), and then differentiating $\phi$ with respect to $s$. Substitution of the expression for $d \phi / d s$ into the general stress rotation equation, Eq. (27), and referring to the constant $-\phi$ stress rotation equation, Eq. (25) and rearranging it to derive

$$
\tan \phi_{e n}=\frac{\tan \phi}{\sqrt{1+2 \tan \phi\left(\Delta I_{D}\right)-\left(\Delta I_{D}\right)^{2}}}
$$

A typical plot for the pressure distribution and mobilised angle of shearing $\phi_{m}$ contour for a conical pile is shown in Figure 10. It can be seen that higher values of $\phi$ are found near the ground surface while lower values are found right beneath the cone. This is because at low confining overburden stress levels in the region away from the pile tip the sand dilates whereas at higher stress levels near the proximity of the cone the dilation is suppressed.

\section{Soil compressibility}

The above variable- $\phi$ analysis has successfully considered the effect of stress-level on $\phi$ but this is insufficient because engineering soils that we encountered are normally compressible. Vesic [36] and Randolph et al. [27] have pointed out the importance of the effects of compressibility on the bearing capacity factors. As explored by Vesic [36], the mode of failure that can be expected in any particular case of a foundation depends on the relative compressibility of the soil in the particular geometrical and loading conditions. If the soil is incompressible and has a finite shearing strength, it will fail in general shear; but if the soil is very compressible for its strength, it will fail in punching shear [36]. The results of the test of model footings in Chattahoochee sand also shows that the mode of failure is somehow associated with foundation depth [36]. In a shallower depth, general shear failure is normally encountered. This mode of failure transformed from general shear into local shear, and finally, punching shear failures as the penetration depth increases.

As there are still no general numerical criteria that can be used to predict the mode of shear failure of soils loaded by foundation, the modified bearing capacity formula, Eq. (29), proposed by Vesic [36] is adopted here:

$$
q_{u(I r)}=q_{u} F_{q c}
$$

where $F_{q c}=$ soil compressibility factor, which can be derived from the analogy of the expansion of cavities.

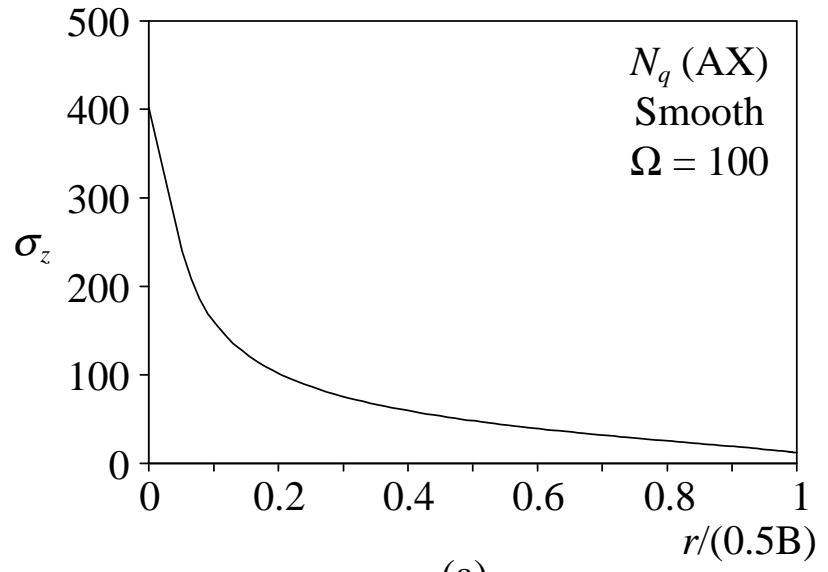

(a)

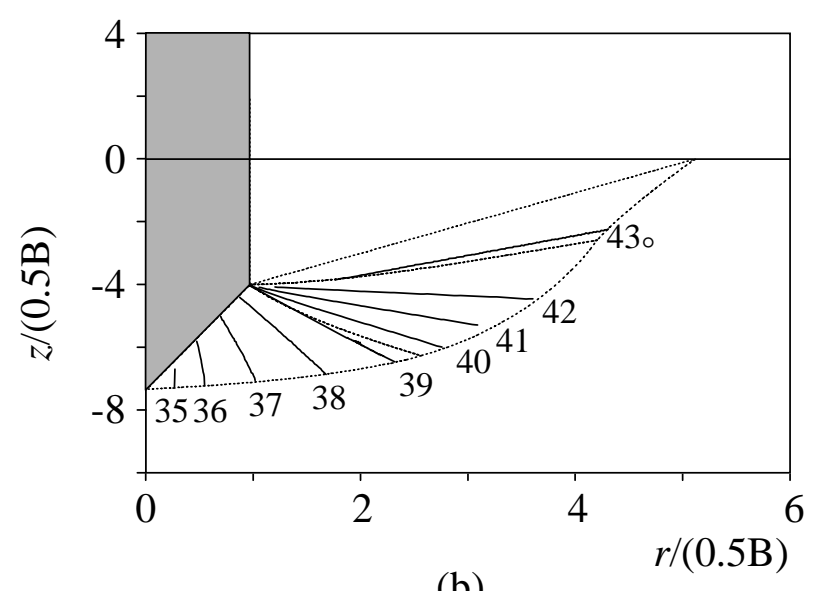

(b)

Fig. 10. A typical (a) pressure distribution; and (b) contour plots of mobilized $\phi$ for a smooth cone in an $N_{q}$ analysis.

To calculate $F_{q c}$, the rigidity index $I_{r}$ must first be calculated using

$$
I_{r}=\frac{G_{50}}{c+\sigma_{v} \tan \phi}
$$

where $G_{50}=$ the secant shear modulus at $50 \%$ peak stress and the cohesion $c$ is taken to be zero for sand. This index varies with the stress level and the characteristic of loading. A high value of $I_{r}$ implies a relatively incompressible material, while a low value implies a relatively compressible material. In addition, to account for the scale effects, the following critical rigidity index has also been proposed by Vesic [36]

$$
I_{r(c r)}=\frac{1}{2}\left\{\exp \left[\left(3.30-0.45 \frac{B}{L}\right) \cot \left(45^{\circ}-\frac{\phi}{2}\right)\right]\right\}
$$

where $B$ and $L=$ width and length of the foundation, 
respectively. If $I_{r}$ is greater than $I_{r(c r)}$, then $F_{q c}=1$. However, if $I_{r}$ is smaller than $I_{r(c r)}$, then

$$
F_{q c}=\exp \left\{\left(-4.4+0.6 \frac{B}{L}\right) \tan \phi+\left[\frac{(3.07 \sin \phi)\left(\log 2 I_{r}\right)}{1+\sin \phi}\right]\right\}
$$

The secant shear modulus at $50 \%$ peak stress of Fontainbleau sand used in the experiment has been deduced from a series of conventional triaxial tests and its shear modulus can be expressed in the following fashion:

$$
G_{50}=\frac{237.5 \sqrt{p^{\prime}}}{e^{4}(1+v)}
$$

where $p^{\prime}=$ mean effective vertical stress, $v=k_{o} /\left(1+k_{o}\right)$ $=$ Poison's ratio, $k_{o}=$ coefficient of lateral earth pressure at rest, and $e=$ void ratio.

\section{COMPARISON WITH CENTRIFUGE TESTS}

Centrifuges have been widely adopted in modeling geotechnical problems. Because, without the expense and delay of doing full-scale tests, the behavior of a foundation can be observed in a soil specimen of known parameters. Here, a $10 \mathrm{~mm}$ diameter miniature penetrometer has been used to model a $700 \mathrm{~mm}$ diameter prototype circular pile, with a $60^{\circ}$ pile shoe, at an acceleration level of $70 \mathrm{~g}$. The test was performed at the center of a $850 \mathrm{~mm}$ diameter container, and the test specimen was prepared by hand pluviating Fontainbleau sand from a single-hole hopper into the container to a height of $350 \mathrm{~mm}$ [4]. It was considered as a normally consolidated specimen as no preloading of the specimen was carried out prior to the test. The inferred value of $d_{50}$ of Fontainbleau sand was $0.181 \mathrm{~mm}$, where $d_{50}$ is the grain diameter at which $50 \%$ of the soil weight is finer. The coefficient of uniformity $\left(d_{60} / d_{10}\right)$ of the sand was found to be 1.69. The average values of maximum and minimum void ratios were 0.55 and 0.92 respectively. Three specimens with final relative densities of $58 \%$, $81 \%$ and $89 \%$ (i.e. dry densities of $1552 \mathrm{~kg} / \mathrm{m}^{3}, 1631 \mathrm{~kg} /$ $\mathrm{m}^{3}$ and $1663 \mathrm{~kg} / \mathrm{m}^{3}$, respectively) were prepared. Particle size effects in the centrifuge were found to be insignificant if the pile diameter to mean particle diameter ratio, $B / d_{50}$, is more than 28 [4]; and in the model the $B / d_{50}$ was 55 .

The model pile was then hydraulically jacked in at a nominal rate of $3.6 \mathrm{~mm} / \mathrm{s}$ under $70 \mathrm{~g}$ in the centrifuge. A load cell located at the pile tip was used to measure the tip resistance and a potentiometer was used to measure the depth of penetration. The tip resistance and depth data are shown in Table 2. The comparison of the tip resistance results from the centrifuge and those based on conventional theories of Eq. (1) and Eq. (2) using the $N_{q}$ factors from variable $-\phi$ analyses are also presented in Table 2 and Figure 11. The $K_{0}=1-\sin \phi$ [16] relation was adopted in Eq. (2). The critical angle of shearing $\phi_{c r i t}$ used was $32^{\circ}[20]$. The mobilized angles of friction (column 5 in Table 2) that corresponded to pile tip level were calculated using equations proposed by Bolton [3], i.e. Eq. (20) and Eq. (21). The roughness of the cone was derived from a series of direct shear box tests by shearing the sand resting on a piece of the cone material, which gave an average value of $\delta / \theta=0.35$. The procedures for the roughness shear box test was essentially the same as the direct shear test for soil except that the bottom half of the shear box was replaced by a piece of $100 \times 100 \mathrm{~mm}^{2}$ cone material instead of soil material.

\begin{tabular}{|c|c|c|c|c|c|c|c|c|}
\hline \multirow{2}{*}{$\mathrm{I}_{\mathrm{D}}(\%)$} & \multirow{2}{*}{$z /(0.5 \mathrm{~B})$} & \multirow{2}{*}{$z(\mathrm{~m})$} & \multirow{2}{*}{$\begin{array}{c}\text { Effective } \\
\text { overburden } \\
\text { stress }(\mathrm{kPa})\end{array}$} & \multirow{2}{*}{$\phi^{\circ}$} & \multirow{2}{*}{$N_{q}$} & \multirow{2}{*}{$\begin{array}{c}\text { Measured } \\
q_{u}(\mathrm{MPa})\end{array}$} & \multicolumn{2}{|c|}{ Predicted $q_{u}(\mathrm{MPa})$} \\
\hline & & & & & & & Eq. (1) & Eq. (2) \\
\hline 58 & 4 & 1.4 & 21.32 & 38.7 & 106.3 & 1.19 & 2.27 & 1.32 \\
\hline 58 & 8 & 2.8 & 42.63 & 37.2 & 172.7 & 3.51 & 7.36 & 4.39 \\
\hline 58 & 12 & 4.2 & 63.95 & 36.2 & 248.0 & 6.88 & 15.86 & 9.61 \\
\hline 81 & 4 & 1.4 & 22.40 & 42.3 & 146.6 & 1.2 & 3.28 & 1.81 \\
\hline 81 & 8 & 2.8 & 44.80 & 40.0 & 225.3 & 3.99 & 10.09 & 5.77 \\
\hline 81 & 12 & 4.2 & 67.20 & 38.7 & 303.0 & 8.25 & 20.36 & 11.87 \\
\hline 81 & 16 & 5.6 & 89.60 & 37.8 & 415.6 & 14.31 & 37.24 & 22.02 \\
\hline 89 & 4 & 1.4 & 22.84 & 43.6 & 164.0 & 1.4 & 3.75 & 2.02 \\
\hline 89 & 8 & 2.8 & 45.68 & 40.9 & 247.1 & 5.2 & 11.29 & 6.36 \\
\hline 89 & 12 & 4.2 & 68.52 & 39.4 & 333.1 & 10.91 & 22.82 & 13.16 \\
\hline 89 & 16 & 5.6 & 91.36 & 38.4 & 421.6 & 17.36 & 38.52 & 22.56 \\
\hline
\end{tabular}

Table 2. Comparison of $q_{u}$ between centrifuge and variable $-\phi$ analysis

Note: $\phi$ were obtained from Eq. (20) and Eq. (21). 
Table 3. Comparison of $q_{u}$ between centrifuge and variable $-\phi$ analysis

\begin{tabular}{rrrrrcrcrrr}
\hline$I_{D}(\%)$ & $z /(0.5 \mathrm{~B})$ & $z(\mathrm{~m})$ & $\phi^{o}$ & $N_{q}$ & $\begin{array}{c}\text { Predicted } q_{c} \\
(\mathrm{MPa}) \\
(=\mathrm{Eq})(2))\end{array}$ & $\begin{array}{c}G_{50} \\
(\mathrm{kPa})\end{array}$ & $F_{q c}$ & $\begin{array}{c}\text { Measured } \\
q_{u}(\mathrm{MPa})\end{array}$ & $\begin{array}{c}\text { Predicted } \\
q_{u(I r)}(\mathrm{MPa}) \\
\left(=F_{q c} \text { Eq. }^{2}\right)\end{array}$ & $\begin{array}{c}\text { Over } \\
\text { Prediction } \\
(\%)\end{array}$ \\
\hline 58 & 4 & 1.4 & 38.7 & 106.3 & 1.32 & 2971 & 0.85 & 1.19 & 1.13 & -5.0 \\
58 & 8 & 2.8 & 37.2 & 172.7 & 4.39 & 3999 & 0.84 & 3.51 & 3.67 & 4.6 \\
58 & 12 & 4.2 & 36.2 & 248.0 & 9.61 & 4817 & 0.83 & 6.88 & 8.00 & 16.3 \\
81 & 4 & 1.4 & 42.3 & 146.6 & 1.81 & 4938 & 0.77 & 1.2 & 1.38 & 15.0 \\
81 & 8 & 2.8 & 40.0 & 225.3 & 5.77 & 6655 & 0.83 & 3.99 & 4.77 & 19.5 \\
81 & 12 & 4.2 & 38.7 & 303.0 & 11.87 & 8020 & 0.85 & 8.25 & 10.07 & 14.7 \\
81 & 16 & 5.6 & 37.8 & 415.6 & 22.02 & 9189 & 0.86 & 14.31 & 18.87 & 31.8 \\
89 & 4 & 1.4 & 43.6 & 164.0 & 2.02 & 6193 & 0.75 & 1.4 & 1.51 & 7.8 \\
89 & 8 & 2.8 & 40.9 & 247.1 & 6.36 & 8353 & 0.85 & 5.2 & 5.39 & 3.6 \\
89 & 12 & 4.2 & 39.4 & 333.1 & 13.16 & 10070 & 0.89 & 10.91 & 11.66 & 6.8 \\
89 & 16 & 5.6 & 38.4 & 421.6 & 22.56 & 11540 & 0.91 & 17.36 & 19.92 & 14.7 \\
\hline
\end{tabular}

Note: $F_{q c}$ and $G_{50}$ were obtained from Eq. (32) and Eq. (33).
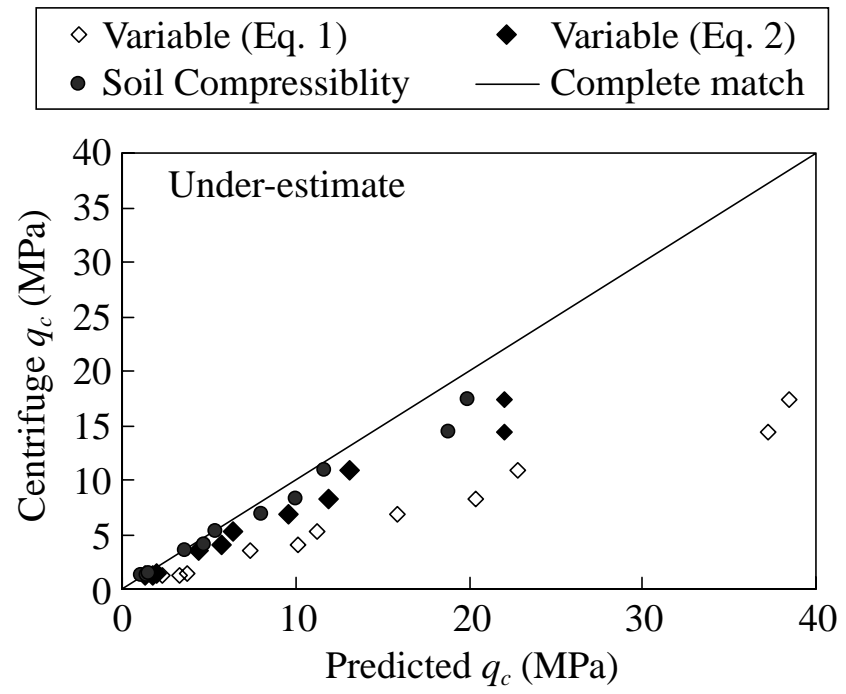

Fig. 11. Predicted $q_{c}$ versus measured $q_{c}$.

From Table 2, it can be seen that the use of Eq. (1) leads to over-estima-tion $(\approx 90-160 \%)$ of the measured tip resistance; the use of Eq. (2) leads to values that are closer to the measured values, with over-estimation of about $11-54 \%$. A better comparison is seen at shallower depth $(z /(0.5 \mathrm{~B})=4)$ than at deeper depth $(z /(0.5 \mathrm{~B})=16)$. This is because the effect of soil compressibility is getting more and more obvious as the overburden stress is getting higher and higher at deeper depth.

After taking the soil compressibility into account, it can be seen from Table 3 and Figure 11 that the analysis has further improved the prediction; this time the over prediction ranges between -5 and $19.5 \%$, with one exception at $31.5 \%$. The result is particularly encouraging for tip resistance ranges between 0 and 12 MPa (Figure 11); thereafter, an over-estimation of tip resistance can still be observed. The tip resistance of 12 $\mathrm{MPa}$ corresponds to a $z /(0.5 \mathrm{~B})=12$ (see Table 3$)$, which is somewhat close to its critical depth value. Meyerhof [23] suggested that the bearing capacity theory in not suitable for estimating tip resistance of a pile below the critical depth and also the tip resistance in a homogeneous sand deposit is easily affected by soil compressibility, crushing, arching, and other factors. Hence, such deviation should be anticipated because only the effect of soil compressibility (not crushing or arching) has been taken into consideration here. However, it should be reasonable to summarize that it is necessary to consider the stress dependent $\phi$ and also the effect of soil compressibility in the bearing capacity calculation for foundations shallower than the critical depth.

\section{CONCLUSION}

The application of the slip line method to estimate the bearing capacity of a shallow wedge foundation/ conical pile has been demonstrated. The angle of friction $\phi$, the depth ratio $Z$, and the surface roughness ratio $\delta / \phi$ affect the end bearing. The constant $-\phi$ method of slip line has also been extended for the case of variable $-\phi$.

The observed reversion of the slip lines during the advancement of the cone clearly showed the generation of the extra rotation of the principal stress (from horizontal direction to vertical direction). This rotation leads to an increase in the bearing capacity value with penetration depth over that of a shallow foundation.

Results of the variable $-\phi$ analysis together with 
the consideration of soil compressibility have been compared with centrifuge test data and it was found that the predictions only differed slightly from the measured values. It thus highlights the need to account for stress variation and soil compressibility.

It is suggested that engineers should define the material strength envelopes appropriate to the material density and stress level and account for surface roughness conditions and soil compressibility to determine the depth dependent bearing capacity factors directly rather than modifying the bearing capacity factors for surface footing as is often done in the current practice.

\section{ACKNOWLEDGEMENTS}

The work presented herein is based on a collaborative research that was initiated at Cambridge University and subsequently completed at National Taipei University of Technology. The second author acknowledges the International Fellowship Award by the US National Science Foundation that enabled the initiation of this collaborative effort with the first author.

\section{REFERENCES}

1. Atkinson, J.H., Foundations and Slopes, McGraw-Hill, UK (1981).

2. De Beer, E.E., "Experimental Determination of the Slope Factors and the Bearing Capacity Factors of Sand," Geotechnique, Vol. 20, No. 4, pp. 387-411 (1970).

3. Bolton, M.D., "The Strength and Dilatancy of Sands," Geotechnique, Vol. 36, No. 1, pp. 65-78 (1986).

4. Bolton, M.D., Gui, M.W., Garnier, J., Corte, J.F., Bagge, G., Laue, J., and Renzi, R., "Centrifuge Modelling of Cone Penetration Tests in Sand," Geotechnique, Vol. 49, No. 4, pp. 543-552 (1999).

5. Bolton, M.D., Gui, M.W., and Phillips, R., "Review of Miniature Soil Probes," Proceedings of 11th Southeast Asia Geotechnical Conference, Singapore, pp. 85-91 (1993).

6. Bolton, M.D. and Lau, C.K., "Vertical Bearing Capacity Factors for Circular and Strip Footings on Mohr-Coulomb Soil," Canadian Geotechnical Journal, Vol. 30, pp. 1024-1033 (1993).

7. Chen, W.F., Limit Analyses and Soil Plasticity, Elsevier, Amsterdam (1975).

8. Cox, A.D., "Axially Symmetry Plastic Deformation in Soils: Indentation Of Ponderable Soils," International Journal of Mechanical Sciences, Vol. 4, pp. 371-380 (1962).

9. Cox, A.D., Eason, G., and Hopkins, H.G., "Axially Symmetric Plastic Deformation in Soils," Philosophical Transaction of the Royal Society of London Series A, Vol. 254, pp. 1-45 (1961).
10. Davis, E.H. and Booker, J.R., "The Bearing Capacity of Strip Footings from the Standpoint of Plasticity Theory," Proceedings of $1^{\text {st }}$ Australian-New Zealand Conference in Geomechanics, Melbourne, pp. 276-282 (1971).

11. Graham, J. and Hovan, J.M., "Stress Characteristics for Bearing Capacity in Sand Using a Critical State Model," Canadian Geotechnical Journal, Vol. 23, pp. 195-202 (1986).

12. Graham, J. and Pollock, D.J., "Scale Dependent Plasticity Analysis for Sand," Civil Engineering \& Public Works Review, Vol. 67, pp. 245-251 (1972).

13. Haar, A. and von Karman, T., "Zur Theorie der Spannungszustaende in Plastichen und Sandartigen Medien," Nachrichten von der Koniglichen Gesellschaft der Wissenschaften, Gottingen, Math.-phys. Klasse, p. 204 (1909).

14. Houlsby, G.T. and Hitchman, R., "Calibration Chamber Tests of a Cone Penetrometer in Sand," Geotechnique, Vol. 38, No. 1, pp. 39-44 (1988).

15. Houlsby, G.T. and Wroth, C.P., "Direct Solution of Plasticity Problems in Soils by the Method of Characteristics," Proceedings of $4^{\text {th }}$ International Conference on Numerical Methods in Geomechanics, Edmonton, Canada, pp. 1059-1071 (1982).

16. Jaky, J., "The Coefficient of Earth Pressure at Rest," Journal of the Union of Hungarian Engineers and Architects, pp. 355-358 (1944).

17. Larkin, L.A., "Theoretical Bearing Capacity of Footings in Plane Strain," Journal of the Soil Mechanics and Foundations Division ASCE, Vol. 94, No. 6, pp. 13471357 (1968).

18. Lau, C.K., "Scale Effects in Tests on Footings," Ph.D. Dissertation, Department of Engineering, University of Cambridge, UK (1988).

19. Lee, S.Y., "Centrifuge Modelling of Cone Penetration Testing in Cohesionless Soils," Ph.D. Dissertation, Department of Engineering, University of Cambridge, UK (1990).

20. Luong, M.P. and Touati, A., "Sols Grenus Sous Fortes Contraintes," Revue Francaix de Geotechnique, Vol. 23. pp. 51-63 (1983).

21. Meyerhof, G.G., "The Ultimate Bearing Capacity of Foundations," Geotechnique, Vol. 2, No. 4, pp. 301-332 (1951).

22. Meyerhof, G.G., "Influence of Roughness of Base and Ground Water Conditions on the Ultimate Bearing Capacity of Foundations," Geotechnique, Vol. 5, No. 3, pp. 227-242 (1955).

23. Meyerhof, G.G., "Bearing Capacity and Settlement of Pile Foundations," Journal of Geotechnical Engineering-ASCE, Vol. 102, No. 3, pp. 197-228 (1976).

24. Nowatzki, E.A., "A Theoretical Assessment of the SPT," Proceedings of $4^{\text {th }}$ Panamerican Conference, San 
Juan, Puerto Rico, pp. 45-61 (1971).

25. Perkins, S.W. and Madsen, C.R., "Bearing Capacity of Shallow Foundations on Sand: a Relative Density Approach," Journal of Geotechnica. and Geoenvironmental Engineering, Vol. 126, No. 6, pp. 521-530 (2000).

26. Prandtl, L., "Uber die Härte Plasticher Körper," Narchrichten von der Königlichen Gesellschaft der Wissenschaften, Gottingen, Math.-phys. Klasse, pp. 7485 (1920).

27. Randolph, M.F., Dolwin, J., and Beck, R., "Design of Driven Piles in Sand," Geotechnique, Vol. 44, No. 3, pp. 427-448 (1994).

28. Reissner, H., "Zum Erddruckproblem," Proceedings of $I^{\text {st }}$ International Conference Applied Mechanics, Delft, Netherlands, pp. 295-311 (1924).

29. Shi, Q., "Centrifugal Modelling of Surface Footings Subjected to Combined Loading," Ph.D. Dissertation, Department of Engineering, University of Cambridge, Cambridge, UK (1988).

30. Shield, R.T., "On the Plastic Flow of Metals under Conditions of Axial Symmetry," Proceedings of the Royal Society of London, Series A, Vol. 233, pp. 267-287 (1955).

31. De Simone, P. and Sapio, G., "End Bearing Capacity of Pile Foundations by Means of Characteristics," Proceedings of 11 th International Conference On Soil Mechanics and Foundation Engineering, San Francisco, LA, Vol. 3, pp. 1373-1376 (1985).

32. Simonini, P., "Analysis of Behavior of Sand Surrounding Pile Tips," Journal of Geotechnical EngineeringASCE, Vol. 122, No. 11, pp. 897-905 (1996).

33. Sokolovski, V.V., Statics of Soil Media. Butterworths, London (1960). (Translated from the 1924 Russian edition.)

34. Taylor, D.W., Fundamentals of Soil Mechanics, John Wiley and Sons, New York (1948).

35. Terzaghi, K., Theoretical Soil Mechanics, John Wiley and Sons, New York (1943).

36. Vesic, A., "Analysis of Ultimate Loads of Shallow Foundations," In Winterkorn, H.F. and Fang, V.N.R. (Ed.), Foundation Engineering Handbook, pp. 121-147 (1973).

37. Vesic, A., Design of Pile Foundations (National Cooperative Highway Research Report 42), Transportation Research Board, Washington DC (1977).

\section{APPENDIX I: NOMENCLATURE}

$\begin{array}{ll}\text { Roman } & \\ B & \text { Diameter or width of foundation } \\ c & \text { Strength intercept (cohesion) } \\ c^{*} & \text { Relative cohesion }\end{array}$

$\begin{array}{ll}I_{D} & \text { Relative density } \\ I_{R} & \text { Dimensionless dilatancy index } \\ K & \text { Ratio of major to minor principal stress } \\ K_{o} & \text { Coefficient of lateral earth pressure at } \\ & \text { rest } \\ M U & \text { Convergence criterion } \\ N_{q}, N_{c} & \text { Dimensionless bearing capacity factors } \\ P c & \text { Aggregate crushing parameter } \\ q_{u} & \text { Measured tip resistance/Calculated ulti- } \\ R & \text { mate bearing capacity } \\ r, \theta, z & \text { Radius ratio } \\ s & \text { Cylindrical polar coordinates } \\ s_{1} & \text { Mean effective stress } \\ Z & \text { Major principal stress passing through a } \\ Z & \text { discontinuity }\end{array}$

$\begin{array}{ll}\text { Greek } & \\ \alpha, \beta & \text { Pair of stress characteristic } \\ \delta & \text { Surface roughness angle } \\ \Delta & \text { An empirical constant } \\ \phi & \text { Internal angle of friction } \\ \phi_{c r i t} & \text { Internal angle of friction } \\ \phi_{e n} & \text { Angle of local strength envelope describ- } \\ & \text { ing the stress characteristic } \\ \phi_{m} & \text { Mobilized angle of friction } \\ \gamma & \text { Self-weight } \\ \lambda & \text { Direction of body weight } \\ \theta & \text { Distortion angle } \\ \sigma_{1} & \text { Major principal stress } \\ \sigma_{3} & \text { Minor principal stress } \\ \sigma_{a}, \tau_{a} & \text { Stresses acting on point } a \\ \sigma_{b}, \tau_{b} & \text { Stresses acting on point } b \\ \sigma_{n} & \text { Normal stress } \\ \sigma_{o} & \text { Arbitrary surcharge } \\ \sigma_{\theta} & \text { Hoop stress } \\ \sigma_{v} & \text { Effective vertical stress } \\ \sigma_{r}, \sigma_{\theta}, \sigma_{z}, \tau_{r z} & \text { Non-zero stress tensor components } \\ \Sigma & \text { Normalized stress } \\ \tau_{f} & \text { Limiting shear stress } \\ \psi & \text { Orientation of principal stress direction } \\ \Omega & \text { A superposition factor } \\ & \end{array}$

